\title{
Living vs. deceased-donor liver transplantation for patients with hepatocellular carcinoma
}

\author{
Kohei Ogawa, Yasutsugu Takada \\ Department of HBP and Breast Surgery, Ehime University, Ehime, Japan \\ Contributions: (I) Conception and design: K Ogawa; (II) Administrative support: Y Takada; (III) Provision of study materials or patients: K Ogawa; (IV) \\ Collection and assembly of data: K Ogawa; (V) Data analysis and interpretation: K Ogawa; (VI) Manuscript writing: All authors; (VII) Final approval \\ of manuscript: All authors. \\ Correspondence to: Kohei Ogawa. Ehime University, Ehime, Japan. Email: ogawa.kohei.vz@ehime-u.ac.jp.
}

\begin{abstract}
With the scarcity of deceased donor liver grafts, living donor liver transplantation (LDLT) is gaining popularity as an alternative to deceased donor liver transplantation (DDLT) for patients with hepatocellular carcinoma (HCC). However, as the evidence of cases of LDLT accumulates, several authors have reported higher HCC recurrence rates after LDLT. The suggested reasons for the higher recurrence rates following LDLT are related to the small-for-size graft in LDLT, surgical procedures that are specific to LDLT, and the fast-track to LDLT. Fast-tracking to LDLT may not allow sufficient time for evaluation of the biological aggressiveness of tumors, which may result in high recurrence rates due to inclusion of patients with more inherently aggressive tumors. Actually, some studies that reported higher recurrence rates with LDLT included a larger number of cases of HCC with microvascular invasion or poorly differentiated HCC. In order to exclude biologically aggressive HCC preoperatively, selection criteria incorporating tumor markers, such as alpha-fetoprotein (AFP) and des-gamma-carboxyprothrombin (DCP), as well as morphological tumor number and size have been proposed. With more reliable selection criteria incorporating biological markers to eliminate biologically aggressive HCC, LDLT can be a viable treatment option for patients with HCC, providing similar recurrence rates as those achieved with DDLT.
\end{abstract}

Keywords: Liver transplantation; living donor; deceased donor; hepatocellular carcinoma (HCC); recurrence

Received: 10 March 2016; Accepted: 07 April 2016; Published: 04 May 2016.

doi: $10.21037 / \operatorname{tgh} .2016 .04 .03$

View this article at: http://dx.doi.org/10.21037/tgh.2016.04.03

\section{Introduction}

Hepatocellular carcinoma (HCC) is the fifth most common cancer worldwide (1), and the third leading cause of cancer-related mortality (2). The management of HCC is improving with the expansion of resection criteria, due to progresses in surgical technique, advanced locoregional therapy, advent of targeted systemic therapies, newer techniques of radiation therapy, and the possibility of liver transplantation (3). Orthotopic liver transplantation (OLT) is the ideal treatment for patients with HCC and cirrhosis, because it can cure not only HCC but also underlying hepatic disease. In Western transplant centers, OLT for patients with HCC have been performed since early in the history of OLT. Early cases of OLT were performed for extensive unresectable tumors. However, the results were extremely dismal before the early 1990s, because of high recurrence rates after OLT $(4,5)$. Since Mazzaferro et al. reported good results with OLT in cases with a single cancerous nodule with a maximal diameter of $5 \mathrm{~cm}$, or up to 3 nodules with a maximal diameter of $3 \mathrm{~cm}$ in 1996 (6), these criteria (the Milan criteria) have been applied for patient selection in Western transplant centers, which has resulted in a dramatic improvement in the results of OLT for patients with HCC. Currently, deceased donor liver transplantation (DDLT) has become widely accepted as the treatment of choice for early HCC. However, the number of deceased donors is far less than the number of potential 
OLT candidates affected with HCC, and up to $30 \%$ of patients develop contraindications to transplantation while waiting for a donor because of tumor progression (7-9). On the other hand, living donor liver transplantation (LDLT) for patients with HCC has developed mainly in Asian countries, including Japan, where deceased donors are seldom available, and results comparable to those of DDLT have been reported (10). The use of LDLT may shorten the waiting time and possibly decrease the waiting list mortality associated with DDLT. Therefore, LDLT for patients with HCC is being accepted worldwide as a solution to donor shortage and waiting list mortality $(11,12)$. However, with the accumulation of cases, higher recurrence rates after LDLT in comparison to DDLT have been reported by several authors (13-17). In this review, we aim to present a current overview of the outcomes of LDLT compared to those of DDLT in patients with HCC, with emphasis on the recurrence of HCC.

\section{Comparative studies of LDLT vs. DDLT for patients with HCC}

LDLT has certain specific features in comparison with DDLT, relating to the graft allocation system, operative procedure, graft size, and graft regeneration after LT. It is still not clear whether these LDLT-specific features could adversely influence the recurrence of HCC. In 2003, Kaihara et al. (10) from Japan reported the results of 56 LDLTs in patients with HCC. The recurrence-free survival rates of all the study patients at 1- and 3-year were $85.4 \%$ and $68.3 \%$, respectively, and those in patients who satisfied the Milan criteria were $87.4 \%$ and $76.2 \%$, respectively. Although these results of LDLT were comparable to the previously reported results of DDLT, further comparative study was needed to clarify the impact of LDLT on recipient outcome compared to DDLT.

In 2004, Gondolesi et al. (18) from the Mount Sinai Hospital reported the results of 36 patients with HCC who underwent LDLT, comparing their results to those of 165 DDLT recipients. They found no significant difference in overall survival (OS) and recurrence-free survival at 1- and 2 -year between LDLT and DDLT recipients $75 \%$ and $60 \%$ vs. $81 \%$ and $70 \%$ OS, and $81 \%$ and $74 \%$ vs. $90 \%$ and $83 \%$ recurrence-free survival). Their study included patients with large HCCs $(>5 \mathrm{~cm})$, and the OS and recurrencefree survival were not statistically significantly different for LDLT and DDLT.

Hwang et al. (19) from Korea reported a comparative study performed at four institutions in 2005. Although there were no gross differences in tumor characteristics between LDLT $(n=237)$ and DDLT $(n=75)$ groups, their results showed significantly better 3-year OS with less perioperative mortality in the LDLT group than the DDLT group $(73.2 \%$ vs. $61.1 \%, \mathrm{P}=0.043)$. There was also no significant difference in recurrence-free survival between the two groups. They evaluated survival according to three different selection criteria [Milan, University of California at San Francisco (UCSF) (20,21), and exclusion of gross major vessel invasion], and found that the survival rates of patients who met these three criteria were excellent for both LDLT and DDLT. However, in the LDLT group, survival of recipients who did not meet these criteria was statistically significantly worse than that of recipients who met the criteria.

In 2009, Di Sandro et al. (22) from Italy reported a comparative study of 25 LDLTs and 154 DDLTs. In this study, patients were selected based on the Milan criteria (80\% LDLT and 69\% DDLT recipients met the Milan criteria), and there were no significant differences in tumor characteristics between the two groups. The overall 3and 5 -year survival rates were $77.3 \%$ and $68.7 \%$ vs. $82.8 \%$ and $76.7 \%$ for LDLT and DDLT recipients, respectively, indicating no significant differences. The recurrence-free survival rates at 3 - and 5-year were also not significantly different between LDLT and DDLT $(95.5 \%$ and $95.5 \%$ vs. $90.5 \%$ and $89.4 \%$, respectively).

In 2010, Li et al. (23) from China also reported the results of 38 LDLTs and 101 DDLTs, with no significant differences in long-term survival (1- and 3-year: $81 \%$ and $53 \%$ vs. $86 \%$ and $50 \%$, respectively, $\mathrm{P}=0.571)$ and recurrence-free survival rates $(71 \%$ and $42 \%$ vs. $76 \%$ and $41 \%$, respectively, $\mathrm{P}=0.787$ ) between LDLT and DDLT.

On the other hand, several studies have also reported worse recurrence rates with LDLT than DDLT.

In 2007, Lo et al. (13) reported the results of 60 cases of OLT, including 43 LDLTs and 17 DDLTs. These 60 cases satisfied the radiological Milan or UCSF criteria. They reported that the cumulative 5 -year recurrence rate of LDLT was $29 \%$, which was significantly higher than that of DDLT (0\%). In the LDLT group, the waiting time for a graft was shorter and graft weight to standard liver weight ratio was lower. LDLT was commonly used for salvage transplantation for recurrence after previous resection or local ablation. Even though the difference did not reach statistical significance, microvascular invasion was more common in the LDLT than DDLT group. Multivariate 
analysis identified salvage transplantation and pathological stage beyond the UCSF criteria as independent predictors of recurrence. The authors speculated that, despite standard radiological selection criteria based on number and size, the higher recurrence rates following LDLT could be attributable to a selection bias for other clinical characters.

In the same year, Fisher et al. (14) also reported the results of 106 patients with HCC who were evaluated for LDLT at nine centers participating in the Adult-to-Adult Living Donor Liver transplantation Cohort Study (A2ALL). Among the 106 patients, 58 received LDLT while 34 received DDLT. The recurrence rate of HCC within 3 years after LDLT was significantly higher as compared to after DDLT ( $29 \%$ vs. $0 \%, \mathrm{P}=0.002$ ). In this report, recurrence occurred in patients with both early and advanced stage HCC in the LDLT group. Although the differences did not reach statistical significance, HCCs in the LDLT group were more likely to have vascular invasion (22\% vs. $10 \%)$ and include more poorly differentiated tumors $(15 \% v s .3 \%)$ than the DDLT group. In 2012, after accumulation of a significant number of cases, Kullik et al. (16) reported updated data on 197 cases (100 LDLT and 97 DDLT). According to this report, the 5-year recurrence rate was significantly higher after LDLT than DDLT, as in their previous report (38\% vs. $11 \%, \mathrm{P}=0.0004)$. Although LDLT recipients were more likely to have tumors that did not satisfy the Milan and UCSF criteria, with vascular invasion and significantly larger tumors than in patients who underwent DDLT, they were less likely to receive locoregional therapy for HCC before the LT. Therefore, they concluded that the higher HCC recurrence rate after LDLT was likely due to more advanced HCC and less liver-directed HCC therapy compared to DDLT.

In 2009, Vakili et al. (15) reported a significantly higher recurrence rate in LDLT than DDLT $(28.6 \%$ vs. $12.1 \%$, $\mathrm{P}<0.05)$. However, microvascular invasion was observed in $46 \%$ of HCCs in the LDLT group in this study. Despite the higher recurrence rate in the LDLT group, 5-year OS was significantly better following LDLT than DDLT due to no perioperative mortality secondary to primary graft complications or non-function in the LDLT group. Therefore, the authors concluded that LDLT should be considered a valuable option for patients with HCC despite the high recurrence rates.

Although these three studies reported higher recurrence rates with LDLT compared to DDLT, the proportion of HCC with microvascular invasion or poorly differentiated HCC was relatively high in the LDLT group in these studies.
One of the advantages of LDLT against DDLT is the shorter waiting time, which might reduce the mortality while on the waiting list for DDLT. On the other hand, such "fast-track" to LDLT might be a cause of the high recurrence rates $(14,24)$. Therefore, to evaluate the effectiveness of LDLT for patients with HCC, the OS after enlisting for LT should be compared. To address this issue, in 2011, Bhangui et al. (25) from France reported the results of a comparative intention-to-treat analysis of recurrence rates and survival outcomes after LDLT and DDLT in patients with HCC. Their study evaluated 183 consecutive patients, including 36 LDLTs and 147 DDLTs, over 9 years. Twenty-seven patients in the DDLT group dropped out from the waiting list, while no patients in the LDLT group dropped out. Recurrence rates were similar between LDLT and DDLT (12.9\% vs. $12.7 \%$ ), and OS rates after enlisting and LT were also comparable. However, transplant recipients who did not satisfy the Milan and UCSF criteria showed a trend toward worse outcomes with LDLT compared to DDLT. Therefore, they mentioned that LDLT for patients who do not meet validated criteria should be proposed with caution.

Sandhu et al. (26) from Toronto reported the results of 58 LDLTs and 287 DDLTs at a single-institution in 2012. To eliminate the selection bias related to the risk of recurrence following LDLT, they offered LDLT to all patients for whom cancer clearance could be obtained despite the inferior vena cava (IVC) being left in situ. They also performed liver biopsy for patients with HCC exceeding the Milan criteria to confirm that the dominant lesion was not poorly differentiated. Patient and tumor characteristics were similar except for the underlying hepatic disease (more cases of hepatitis $\mathrm{C}$ virus infection and fewer of hepatitis $\mathrm{B}$ virus infection and alcoholic liver disease in LDLT) and waiting period from enlisting to transplant (significantly shorter with LDLT) between the two groups. OS was not significantly different between the two groups (1- and 5-year survival: $91.3 \%$ and $75.2 \%$ in LDLT vs. $90.5 \%$ and $74.6 \%$ in DDLT). The recurrence rates were also similar (1- and 5-year rates: $8.8 \%$ and $15.4 \%$ in LDLT vs. $7.5 \%$ and $17.0 \%$ in DDLT). They concluded that LDLT and DDLT provide similarly low recurrence rates and high survival rates for the treatment of HCC in well-matched cohorts of LDLT and DDLT recipients.

\section{Meta-analysis}

In 2012, a meta-analysis of comparative studies of LDLT 
vs. DDLT for HCC was reported by Liang et al. (27) from China. They selected seven controlled clinical trials with 1,310 participants for the analysis. Their analysis showed no significant differences in patient survival, recurrence-free survival and recurrence rates between LDLT and DDLT. For patients who did not meet the Milan criteria, however, LDLT recipients had a greater 1 -year recurrence rate than DDLT recipients.

On the other hand, in 2013, Grant et al. (28) from Toronto selected 16 studies on this subject for systematic review, 12 of which satisfied the eligibility criteria for recurrence-free survival, including 633 LDLT and 1,232 DDLT cases. The analysis suggested that recurrence-free survival was worse after LDLT compared to DDLT, with a combined hazard ratio of 1.59 [95\% confidence interval (CI): $\left.1.02-2.49 ; \mathrm{I}^{2}=50.07 \%\right]$. However, it was difficult to evaluate the risk of bias caused by differences in tumor biology because the patient characteristics were poorly reported. Therefore, they concluded that further research is needed to determine whether the poorer recurrence-free survival in LDLT compared to DDLT was due to study biases or to true biological differences.

\section{Recent reports}

In 2014, Park et al. (17) reported the results of 216 OLT recipients with HCC who met the UCSF criteria (166 LDLT and 50 DDLT). Recurrence-free survival was significantly lower with LDLT compared to DDLT (88.6\% and $80.7 \%$ vs. $96.0 \%$ and $94.0 \%$ at $1-$ and 5 -year, $\mathrm{P}=0.045$ ). A higher proportion of patients with microvascular invasion underwent LDLT (41.4\% vs. 20.0\%). However, even after adjustment for microvascular invasion, LDLT was an independent risk factor for recurrence. They also mentioned about the high recurrence rate of LDLT using small living donor grafts compared to DDLT.

On the other hand, in 2014, Akamatsu et al. published the review entitled 'Living-donor vs deceased-donor liver transplantation for patients with hepatocellular carcinoma'. In this revew, they reported their original data about the association of graft liver regeneration with HCC recurrence. Their data suggested that there was no differences in the regeneration rate of graft liver between the recipients with HCC recurrence and those without recurrence (29).

In 2015, Ninomiya et al. (30) reported a retrospective analysis between patients with HCC who underwent LDLT in a Japanese institute and those who underwent DDLT in an institute in the United States. In these settings, there was no difference in 5-year recurrence rate between the LDLT and DDLT groups (14.8\% vs. 19.0\%, $\mathrm{P}=0.638$ ), although OS was better in the LDLT group $(84.2 \%$ vs. $63.5 \%, \mathrm{P}<0.0001)$. They rejected the hypothesis that tumor growth was induced by regeneration of small-for-size grafts, because they preferentially used smaller left-sided partial grafts for LDLT. They concluded that recurrence rates were comparable between the two centers where LDLT or DDLT was performed as the first treatment choice for unresectable HCC.

Another comparative study of LDLT and DDLT for patients with HCC using expanded selection criteria was reported by Chen et al. (31) from China. In this study, they compared the results of 66 LDLTs and 163 DDLTs, after excluding 26 patients who died during the perioperative period, in terms of their compliance with the Milan criteria, UCSF criteria, up-to-seven criteria (32), Hangzhou criteria (33), and Chengdu criteria (34). The overall recurrence-free survival and OS rates of the Milan criteriabased recipients, UCSF recipients, up-to-seven recipients, Hangzhou recipients, and Chengdu recipients after LDLT and DDLT were all similar.

A multi-center data analysis from China was also reported in the same year (35). They analyzed the data of 389 LDLTs and 6,471 DDLTs from 81 centers over a 10 -year period retrospectively using the large, multi-center database of the China Liver Transplant Registry. The 1-, 3-, and 5-year OS rates were, respectively, $86.79 \%, 70.16 \%$, and $66.31 \%$ after LDLT, and $74.2 \%, 54.21 \%$, and $46.97 \%$ after DDLT $(\mathrm{P}<0.001)$. The 1-, 3-, and 5-year recurrence-free survival rates were, respectively, $78.46 \%, 63.68 \%$, and $61.63 \%$ after LDLT, and $65.65 \%, 48.61 \%$, and $41.87 \%$ after DDLT $(\mathrm{P}<0.001)$. They concluded that there were no disadvantages with LDLT compared to DDLT regarding OS and recurrence-free survival.

Comparative studies between LDLT and DDLT for patients with HCC, including reports that are not mentioned above, are summarized in Table 1.

\section{Hypothesized mechanism of increased risk of recurrence with LDLT than DDLT}

The inferior results of LDLT have been attributed to growth factors and cytokines released during acute graft injury, and subsequent graft regeneration resulting from the small-for-size graft in LDLT (36-39). Acute phase small-forsize graft injury, characterized by hepatic sinusoidal damage, is caused by excessive portal flow and transient portal 


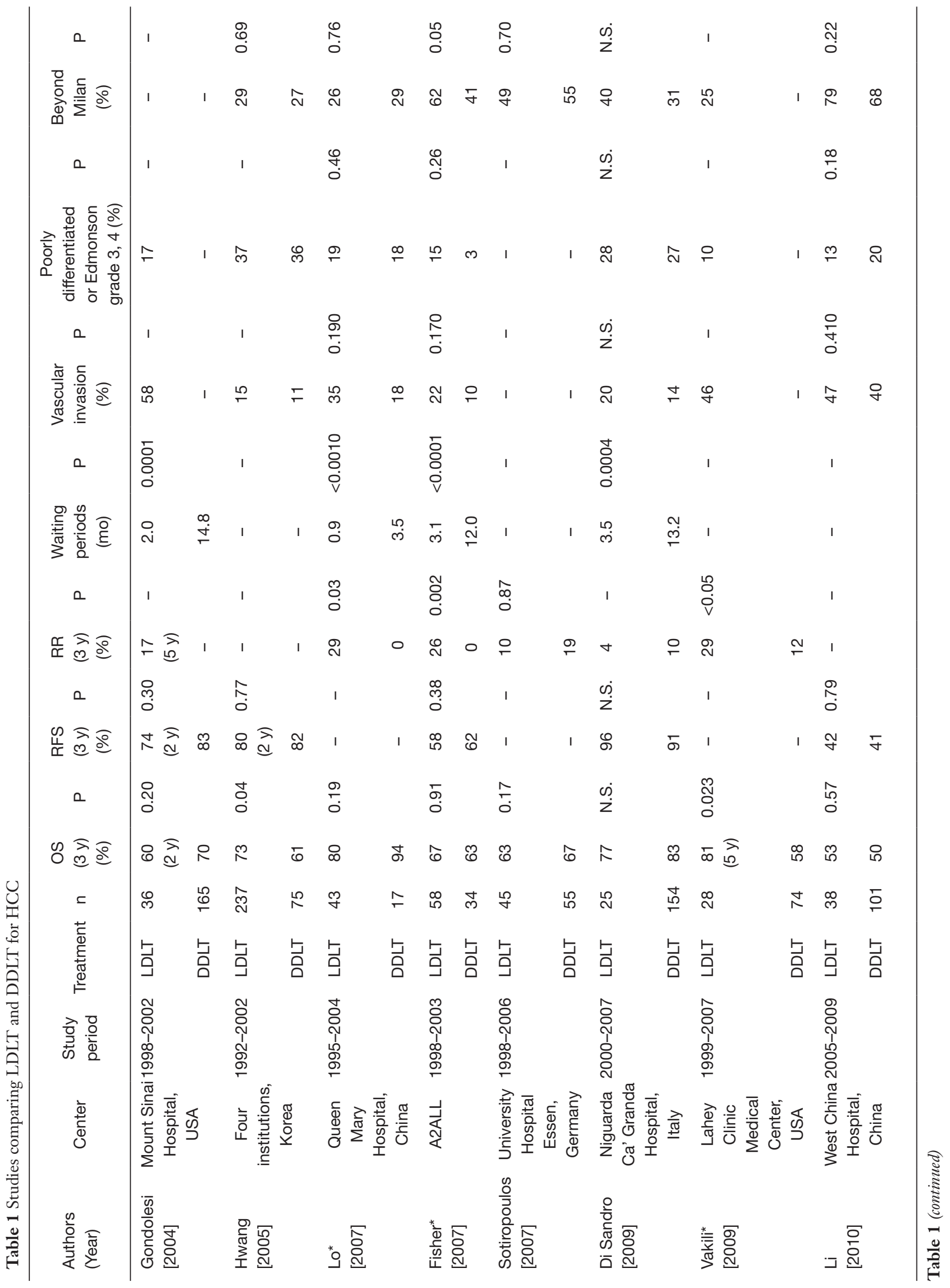




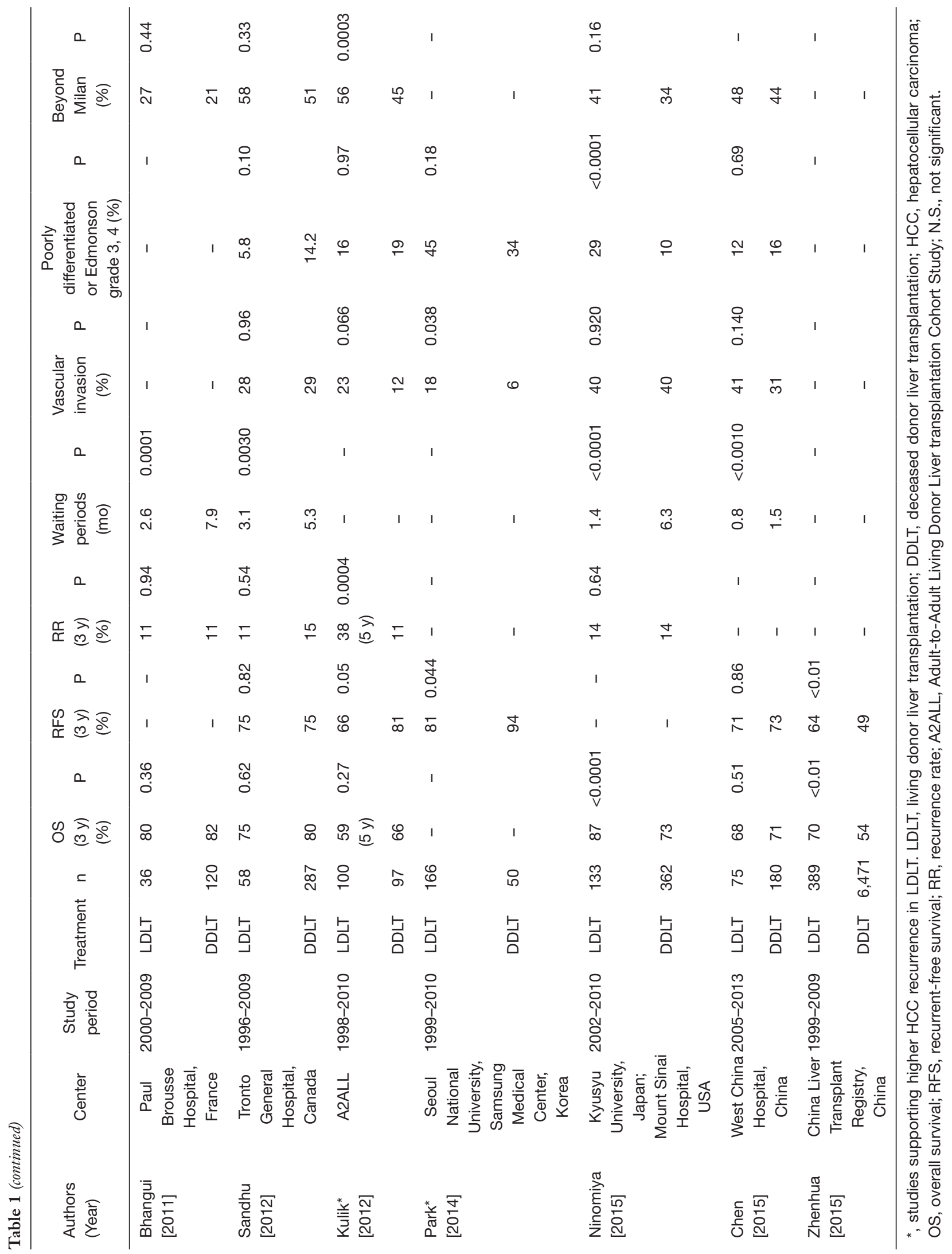


hypertension. Man et al. reported that significant activation of cell signaling pathways caused by this graft injury leads to cell adhesion, angiogenesis, cell invasion, and migration, which promoted tumor growth and metastasis after LT in an animal study (38). Rapid liver regeneration after small-for-size LDLT could also provide a more favorable environment for tumor growth and metastasis, as Shi et al. reported, since HCC tumors developing after hepatectomy display growth rates and malignant transformations related to the size of the partial hepatectomy (39).

Certain technical aspects of LDLT have also been implicated as the cause for the higher recurrence $(14,22,23)$. During total hepatectomy in LDLT, the recipient's IVC is totally isolated from the HCC liver. If the HCC is located near the IVC, this procedure might lead to tumor remnants being left behind. The necessity for leaving a longer hepatic artery, portal vein, and bile duct for anastomosis in LDLT recipients might also lead to residual tumor remnants. Rotation and more manipulation of the native liver with HCC may also increase the risk of seeding tumor cells into the circulation. On the other hand, total hepatectomy with IVC excision in DDLT seems to be oncologically more favorable.

Finally, the fast-track effect is thought to be a possible reason for the inferior results of LDLT $(14,24)$. During the long waiting periods of DDLT, patients are usually treated with locoregional therapy, such as transarterial chemo-embolization (TACE) and radiofrequency ablation (RFA). During this period, several patients with biologically aggressive HCC drop out of the waiting list due to tumor progression. On the other hand, LDLT has the advantage of shorter waiting periods than DDLT, which might decrease the mortality on the waiting list. However, such fast-track to LDLT may not allow adequate time for evaluation of the biological aggressiveness of tumors, which may result in high recurrence rates due to the inclusion of patients with inherently more aggressive tumor biology.

\section{Biomarkers to predict biologically aggressive HCC}

In Western countries, the characteristics of patients with HCC are different between LDLT and DDLT due to a possible bias in terms of treatment selection. For example, especially in the United States where the organ allocation system gives higher priority to patients with HCC who satisfy the Milan criteria (40), such patients are preferentially treated with DDLT (14). Moreover, the "fast- track" to LDLT may result in inclusion of patients with biologically aggressive HCCs. As a result, patients who undergo LDLT often have more advanced tumors and more frequently have biologically aggressive tumors. These are some of the reasons for the higher recurrence rates of HCC after LDLT in some previous reports (13-16).

In order to resolve this problem, the biological aggressiveness of HCC should be accurately assessed preoperatively. Morphological assessment alone, such as the number and size of the tumor, might be insufficient to exclude biologically aggressive tumors, since, as reported by many previous comparative studies, many cases in the LDLT group that satisfied the Milan or UCSF criteria also had microvascular invasion and poorly differentiated HCCs. Therefore, recently, many transplant institutes are using selection criteria that incorporate tumor markers, such as alpha-fetoprotein (AFP) and des-gamma-carboxyprothrombin (DCP), as well as tumor number and size to exclude biologically aggressive HCC preoperatively (33,41-49). Takada et al. reported that DCP $>400 \mathrm{mAU} / \mathrm{mL}$ was an independent risk factor of HCC recurrence after LDLT, and proposed expanded selection criteria, the Kyoto criteria, which include tumor number $\leq 10$, maximal diameter of each tumor $\leq 5 \mathrm{~cm}$, and DCP levels of $\leq 400 \mathrm{mAU} / \mathrm{mL}(41,42)$. Kaido et al. reported excellent OS and low recurrence rates in patients who met the Kyoto criteria (5-year OS: $82 \%$, and 5 -year recurrence rate: $4 \%$ ) (50). The selection criteria of LT for patients with HCC, including the criteria incorporating tumor markers, are summarized in Table 2.

Other biological markers, such as fludeoxyglucosepositron emission tomography (FDG-PET) and the neutrophil-to-lymphocyte ratio (the ratio of neutrophil count to lymphocyte count: NLR) are also used to assess biological aggressiveness (51-54). Kornberg et al. (51) reported that tumor uptake of ${ }^{18} \mathrm{~F}-\mathrm{FDG}$ on preoperative PET scans is significantly associated with tumor grade as well as with microvascular invasion on explant pathology in recipients with HCC, and Lee et al. (52) reported that ${ }^{18} \mathrm{~F}-\mathrm{FDG}-\mathrm{PET}$ is a significant predictor of tumor recurrence in liver transplantation. NLR, which is a simple index of systemic inflammation, has been reported to be associated with prognosis in patients with various kinds of malignancies, including HCC $(53,54)$.

Such biological markers, in combination with morphological tumor size and number, might assist in eliminating biologically aggressive HCC preoperatively, 
Table 2 Selection criteria of LT for patients with HCC

\begin{tabular}{|c|c|c|c|}
\hline Authors & Criteria or institute & Year & Variables \\
\hline \multicolumn{4}{|c|}{ Criteria based on morphology of $\mathrm{HCC}$} \\
\hline Mazzaferro (6) & Milan & 1996 & (I) Number $\leq 3$, size $\leq 3 \mathrm{~cm}$; (II) single tumor, size $\leq 5 \mathrm{~cm}$ \\
\hline Yao (20) & UCSF & 2001 & $\begin{array}{l}\text { (l) Single tumor, size } \leq 6.5 \mathrm{~cm} \text {; (II) number } \leq 3 \text {, size }<4.5 \mathrm{~cm} \text {, total tumor } \\
\text { diameter } \leq 8 \mathrm{~cm}\end{array}$ \\
\hline \multicolumn{4}{|c|}{ Criteria incorporating serum tumor marker } \\
\hline Takada (41) & Kyoto & 2007 & Number $\leq 10$, size $\leq 5 \mathrm{~cm}, \mathrm{DCP} \leq 400 \mathrm{mAU} / \mathrm{mL}$ \\
\hline Soejima (43) & Kyusyu & 2007 & Size $\leq 5 \mathrm{~cm}, \mathrm{DCP} \leq 300 \mathrm{mAU} / \mathrm{mL}$ \\
\hline Duvoux (48) & $\begin{array}{l}\text { Liver Transplantation } \\
\text { French Study Group }\end{array}$ & 2012 & $\begin{array}{l}\text { (I) Number } \leq 3 \text {, size } \leq 6 \mathrm{~cm} \text {, AFP } \leq 100 \mathrm{ng} / \mathrm{mL} \text {; (II) number } \geq 4 \text {, size } \leq 3 \mathrm{~cm} \text {, AFP } \\
\leq 100 \mathrm{ng} / \mathrm{mL}\end{array}$ \\
\hline Shindoh (49) & Tokyo & 2014 & $\begin{array}{l}\text { (I) Number } \leq 5 \text {, size } \leq 5 \mathrm{~cm} \text {, and AFP } \leq 250 \mathrm{ng} / \mathrm{mL} \text { or DCP } \leq 450 \mathrm{mAU} / \mathrm{mL} \text {; (II) } \\
\text { AFP } \leq 250 \mathrm{ng} / \mathrm{mL} \text { and DCP } \leq 450 \mathrm{mAU} / \mathrm{mL}\end{array}$ \\
\hline
\end{tabular}

LT, liver transplantation; HCC, hepatocellular carcinoma; DCP, des-gamma-carboxyprothrombin; AFP, alpha-fetoprotein.

which will lead to lower recurrence and better recurrencefree survival after LDLT (55).

\section{Conclusions}

LDLT can lead to a decrease in mortality while on the liver transplant waiting list, and provide better quality grafts due to minimal fatty changes and less cold ischemic time compared to DDLT. Although the higher recurrence rates in LDLT reported by several authors cannot be ignored, low recurrence rates can be achieved with LDLT as long as biologically aggressive HCCs are eliminated. Therefore, with more reliable selection criteria incorporating biological markers to eliminate biologically aggressive HCCs, LDLT can be a viable treatment option for patients with HCC, providing similar recurrence rates as those achieved with DDLT.

\section{Acknowledgements}

None.

\section{Footnote}

Conflicts of Interest: The authors have no conflicts of interest to declare.

\section{References}

1. Ferlay J, Soerjomataram I, Dikshit R, et al. Cancer incidence and mortality worldwide: sources, methods and major patterns in GLOBOCAN 2012. Int J Cancer 2015;136:E359-86.

2. Willatt JM, Francis IR, Novelli PM, et al. Interventional therapies for hepatocellular carcinoma. Cancer Imaging 2012;12:79-88.

3. Dhir M, Melin AA, Douaiher J, et al. A Review and Update of Treatment Options and Controversies in the Management of Hepatocellular Carcinoma. Ann Surg 2016. [Epub ahead of print].

4. O'Grady JG, Polson RJ, Rolles K, et al. Liver transplantation for malignant disease. Results in 93 consecutive patients. Ann Surg 1988;207:373-9.

5. Ringe B, Pichlmayr R, Wittekind C, et al. Surgical treatment of hepatocellular carcinoma: experience with liver resection and transplantation in 198 patients. World J Surg 1991;15:270-85.

6. Mazzaferro V, Regalia E, Doci R, et al. Liver transplantation for the treatment of small hepatocellular 
carcinomas in patients with cirrhosis. N Engl J Med 1996;334:693-9.

7. Yao FY, Bass NM, Nikolai B, et al. Liver transplantation for hepatocellular carcinoma: analysis of survival according to the intention-to-treat principle and dropout from the waiting list. Liver Transpl 2002;8:873-83.

8. Graziadei IW, Sandmueller H, Waldenberger P, et al. Chemoembolization followed by liver transplantation for hepatocellular carcinoma impedes tumor progression while on the waiting list and leads to excellent outcome. Liver Transpl 2003;9:557-63.

9. Fisher RA, Maluf D, Cotterell AH, et al. Non-resective ablation therapy for hepatocellular carcinoma: effectiveness measured by intention-to-treat and dropout from liver transplant waiting list. Clin Transplant 2004;18:502-12.

10. Kaihara S, Kiuchi T, Ueda M, et al. Living-donor liver transplantation for hepatocellular carcinoma. Transplantation 2003;75:S37-40.

11. Sarasin FP, Majno PE, Llovet JM, et al. Living donor liver transplantation for early hepatocellular carcinoma: A lifeexpectancy and cost-effectiveness perspective. Hepatology 2001;33:1073-9.

12. Lo CM, Fan ST, Liu CL, et al. The role and limitation of living donor liver transplantation for hepatocellular carcinoma. Liver Transpl 2004;10:440-7.

13. Lo CM, Fan ST, Liu CL, et al. Living donor versus deceased donor liver transplantation for early irresectable hepatocellular carcinoma. Br J Surg 2007;94:78-86.

14. Fisher RA, Kulik LM, Freise CE, et al. Hepatocellular carcinoma recurrence and death following living and deceased donor liver transplantation. Am J Transplant 2007;7:1601-8.

15. Vakili K, Pomposelli JJ, Cheah YL, et al. Living donor liver transplantation for hepatocellular carcinoma: Increased recurrence but improved survival. Liver Transpl 2009;15:1861-6.

16. Kulik LM, Fisher RA, Rodrigo DR, et al. Outcomes of living and deceased donor liver transplant recipients with hepatocellular carcinoma: results of the A2ALL cohort. Am J Transplant 2012;12:2997-3007.

17. Park MS, Lee KW, Suh SW, et al. Living-donor liver transplantation associated with higher incidence of hepatocellular carcinoma recurrence than deceased-donor liver transplantation. Transplantation 2014;97:71-7.

18. Gondolesi GE, Roayaie S, Muñoz L, et al. Adult living donor liver transplantation for patients with hepatocellular carcinoma: extending UNOS priority criteria. Ann Surg 2004;239:142-9.

19. Hwang S, Lee SG, Joh JW, et al. Liver transplantation for adult patients with hepatocellular carcinoma in Korea: comparison between cadaveric donor and living donor liver transplantations. Liver Transpl 2005;11:1265-72.

20. Yao FY, Ferrell L, Bass NM, et al. Liver transplantation for hepatocellular carcinoma: expansion of the tumor size limits does not adversely impact survival. Hepatology 2001;33:1394-403.

21. Yao FY, Xiao L, Bass NM, et al. Liver transplantation for hepatocellular carcinoma: validation of the UCSFexpanded criteria based on preoperative imaging. Am J Transplant 2007;7:2587-96.

22. Di Sandro S, Slim AO, Giacomoni A, et al. Living donor liver transplantation for hepatocellular carcinoma: long-term results compared with deceased donor liver transplantation. Transplant Proc 2009;41:1283-5.

23. Li C, Wen TF, Yan LN, et al. Outcome of hepatocellular carcinoma treated by liver transplantation: comparison of living donor and deceased donor transplantation. Hepatobiliary Pancreat Dis Int 2010;9:366-9.

24. Kulik L, Abecassis M. Living donor liver transplantation for hepatocellular carcinoma. Gastroenterology 2004;127:S277-82.

25. Bhangui P, Vibert E, Majno $\mathrm{P}$, et al. Intention-totreat analysis of liver transplantation for hepatocellular carcinoma: living versus deceased donor transplantation. Hepatology 2011;53:1570-9.

26. Sandhu L, Sandroussi C, Guba M, et al. Living donor liver transplantation versus deceased donor liver transplantation for hepatocellular carcinoma: comparable survival and recurrence. Liver Transpl 2012;18:315-22.

27. Liang $W, W u L$, Ling $X$, et al. Living donor liver transplantation versus deceased donor liver transplantation for hepatocellular carcinoma: a meta-analysis. Liver Transpl 2012;18:1226-36.

28. Grant RC, Sandhu L, Dixon PR, et al. Living vs. deceased donor liver transplantation for hepatocellular carcinoma: a systematic review and meta-analysis. Clin Transplant 2013;27:140-7.

29. Akamatsu N, Sugawara Y, Kokudo N. Living-donor vs deceased-donor liver transplantation for patients with hepatocellular carcinoma. World J Hepatol 2014;6:626-31.

30. Ninomiya M, Shirabe K, Facciuto ME, et al. Comparative study of living and deceased donor liver transplantation as a treatment for hepatocellular carcinoma. J Am Coll Surg 2015;220:297-304.e3.

31. Chen LP, Li C, Wen TF, et al. Can living donor liver transplantation offer similar outcomes to deceased donor liver transplantation using expanded selection criteria for hepatocellular carcinoma? Pak J Med Sci 2015;31:763-9.

32. Mazzaferro V, Llovet JM, Miceli R, et al. Predicting 
survival after liver transplantation in patients with hepatocellular carcinoma beyond the Milan criteria: a retrospective, exploratory analysis. Lancet Oncol 2009; 10:35-43.

33. Zheng SS, Xu X, Wu J, et al. Liver transplantation for hepatocellular carcinoma: Hangzhou experiences. Transplantation 2008;85:1726-32.

34. Li J, Yan LN, Yang J, et al. Indicators of prognosis after liver transplantation in Chinese hepatocellular carcinoma patients. World J Gastroenterol 2009;15:4170-6.

35. Hu Z, Qian Z, Wu J, et al. Clinical outcomes and risk factors of hepatocellular carcinoma treated by liver transplantation: A multi-centre comparison of living donor and deceased donor transplantation. Clin Res Hepatol Gastroenterol 2015. [Epub ahead of print].

36. Man K, Fan ST, Lo CM, et al. Graft injury in relation to graft size in right lobe live donor liver transplantation: a study of hepatic sinusoidal injury in correlation with portal hemodynamics and intragraft gene expression. Ann Surg 2003;237:256-64.

37. Efimova EA, Glanemann M, Liu L, et al. Effects of human hepatocyte growth factor on the proliferation of human hepatocytes and hepatocellular carcinoma cell lines. Eur Surg Res 2004;36:300-7.

38. Man K, Lo CM, Xiao JW, et al. The significance of acute phase small-for-size graft injury on tumor growth and invasiveness after liver transplantation. Ann Surg 2008;247:1049-57.

39. Shi JH, Huitfeldt HS, Suo ZH, et al. Growth of hepatocellular carcinoma in the regenerating liver. Liver Transpl 2011;17:866-74.

40. Schwartz M. Liver transplantation in patients with hepatocellular carcinoma. Liver Transpl 2004;10:S81-5.

41. Takada Y, Ito T, Ueda M, et al. Living donor liver transplantation for patients with HCC exceeding the Milan criteria: a proposal of expanded criteria. Dig Dis 2007;25:299-302.

42. Ito T, Takada Y, Ueda M, et al. Expansion of selection criteria for patients with hepatocellular carcinoma in living donor liver transplantation. Liver Transpl 2007;13:1637-44.

43. Soejima Y, Taketomi A, Yoshizumi T, et al. Extended indication for living donor liver transplantation in patients with hepatocellular carcinoma. Transplantation 2007;83:893-9.

44. Taketomi A, Sanefuji K, Soejima Y, et al. Impact of desgamma-carboxy prothrombin and tumor size on the recurrence of hepatocellular carcinoma after living donor liver transplantation. Transplantation 2009;87:531-7.

45. Todo S, Furukawa H, Tada M, et al. Extending indication: role of living donor liver transplantation for hepatocellular carcinoma. Liver Transpl 2007;13:S48-54.

46. Yang SH, Suh KS, Lee HW, et al. A revised scoring system utilizing serum alphafetoprotein levels to expand candidates for living donor transplantation in hepatocellular carcinoma. Surgery 2007;141:598-609.

47. Toso C, Asthana S, Bigam DL, et al. Reassessing selection criteria prior to liver transplantation for hepatocellular carcinoma utilizing the Scientific Registry of Transplant Recipients database. Hepatology 2009;49:832-8.

48. Duvoux C, Roudot-Thoraval F, Decaens T, et al. Liver transplantation for hepatocellular carcinoma: a model including $\alpha$-fetoprotein improves the performance of Milan criteria. Gastroenterology 2012;143:986-94.e3; quiz e14-5.

49. Shindoh J, Sugawara Y, Nagata R, et al. Evaluation methods for pretransplant oncologic markers and their prognostic impacts in patient undergoing living donor liver transplantation for hepatocellular carcinoma. Transpl Int 2014;27:391-8.

50. Kaido T, Ogawa K, Mori A, et al. Usefulness of the Kyoto criteria as expanded selection criteria for liver transplantation for hepatocellular carcinoma. Surgery 2013;154:1053-60.

51. Kornberg A, Freesmeyer M, Bärthel E, et al. 18F-FDGuptake of hepatocellular carcinoma on PET predicts microvascular tumor invasion in liver transplant patients. Am J Transplant 2009;9:592-600.

52. Lee S, Ahn C, Ha T, et al. Liver transplantation for hepatocellular carcinoma: Korean experience. J Hepatobiliary Pancreat Sci 2010;17:539-47.

53. Gomez D, Farid S, Malik HZ, et al. Preoperative neutrophil-to-lymphocyte ratio as a prognostic predictor after curative resection for hepatocellular carcinoma. World J Surg 2008;32:1757-62.

54. Mano Y, Shirabe K, Yamashita Y, et al. Preoperative neutrophil-to-lymphocyte ratio is a predictor of survival after hepatectomy for hepatocellular carcinoma: a retrospective analysis. Ann Surg 2013;258:301-5.

55. Takada Y, Tohyama T, Watanabe J. Biological markers of hepatocellular carcinoma for use as selection criteria in liver transplantation. J Hepatobiliary Pancreat Sci 2015;22:279-86.

doi: $10.21037 / \operatorname{tgh} .2016 .04 .03$

Cite this article as: Ogawa K, Takada Y. Living vs. deceaseddonor liver transplantation for patients with hepatocellular carcinoma. Transl Gastroenterol Hepatol 2016;1:35. 